\title{
Stimuli-responsive Ferrogele zur Umweltüberwachung von Schwermetallen
}

\author{
Alice Mieting, Yi-Pin Wei, Daniela Franke, Margarita Günther, Gerald Gerlach \\ Institut für Festkörperelektronik, TU Dresden, Dresden, Deutschland \\ Kontakt: alice.mieting@tu-dresden.de
}

\section{Einleitung}

Für eine schnelle Bestimmung von Schadstoffkonzentrationen schwermetallbelasteter Gewässer direkt am Ort der Entstehung ist bislang keine kostengünstige Sensortechnik verfügbar [1]. Eine Lösung zur Etablierung einer effizienteren vor-Ort-Analytik könnte die Entwicklung eines Schwermetall-sensitiven Materials für die Anwendung in einem Drucksensor sein.

Ferrogele sind dafür ein vielversprechendes Material. Sie basieren auf einem hydrophilen Polymernetzwerk inkorporiert mit Eisenoxidpartikeln. Die enthaltenen Eisenoxidpartikel besitzen eine hohe Adsorption gegenüber Schwermetallen [2], [3] und das Polymernetzwerk kann aufgrund seiner viskoelastischen Eigenschaften in wässrigen Umgebungen das Volumen ändern [4]. Die resultierende Stimuli-responsive Volumenänderung kann schließlich über einen piezoresistiven Sensor in ein elektronisches Ausgangssignal übertragen werden [5].

In diesem Beitrag wird die Herstellung von Ferrogelen mittels nasschemischer in-situ Fällung von Eisenoxidpartikeln in Hydrogelen sowie die Charakte- risierung der resultierenden Quelleigenschaften präsentiert. Dabei soll der Einfluss der folgenden Synthesebedingungen auf die Quelleigenschaften der Ferrogele untersucht werden:

1) Konzentration der Fällungsreagenzien und

2) Struktur des Hydrogels als Templat mit lonenkoordinierenden Eigenschaften.

In Abb. 1A ist das Schema zur Herstellung der Ferrogele mit den verwendeten Fällungsreagenzien zur stöchiometrischen Bildung von Magnetit $\left(\mathrm{Fe}^{\mathrm{II}}\left(\mathrm{Fe}^{\mathrm{III}}\right)_{2} \mathrm{O}_{4}\right)$ dargestellt. Um den Einfluss der gebildeten Menge an Eisenoxid auf die sensitiven Quelleigenschaften der Ferrogele zu bestimmen, werden Verdünnungsstufen des Fällungsansatzes angewendet. Zur Untersuchung der Templatstruktur wird ein nichtionisches Polyacrylamid Hydrogel $(\mathrm{P}(\mathrm{AAm}))$ und ein anionisches Copolymer mit Natriumacrylatgruppen ( $\mathrm{P}(\mathrm{NaAc}-\mathrm{Co}-\mathrm{AAm})$ ) verwendet.

Die Quelleigenschaften der Ferrogele werden in Abhängigkeit von der lonenstärke und des pH-Wertes sowie hinsichtlich der Quellkinetik charakterisiert. Anhand der Ergebnisse soll die potenzielle Anwendbarkeit der Ferrogele als Stimuli-sensitives Material für Drucksensoren gezeigt werden.

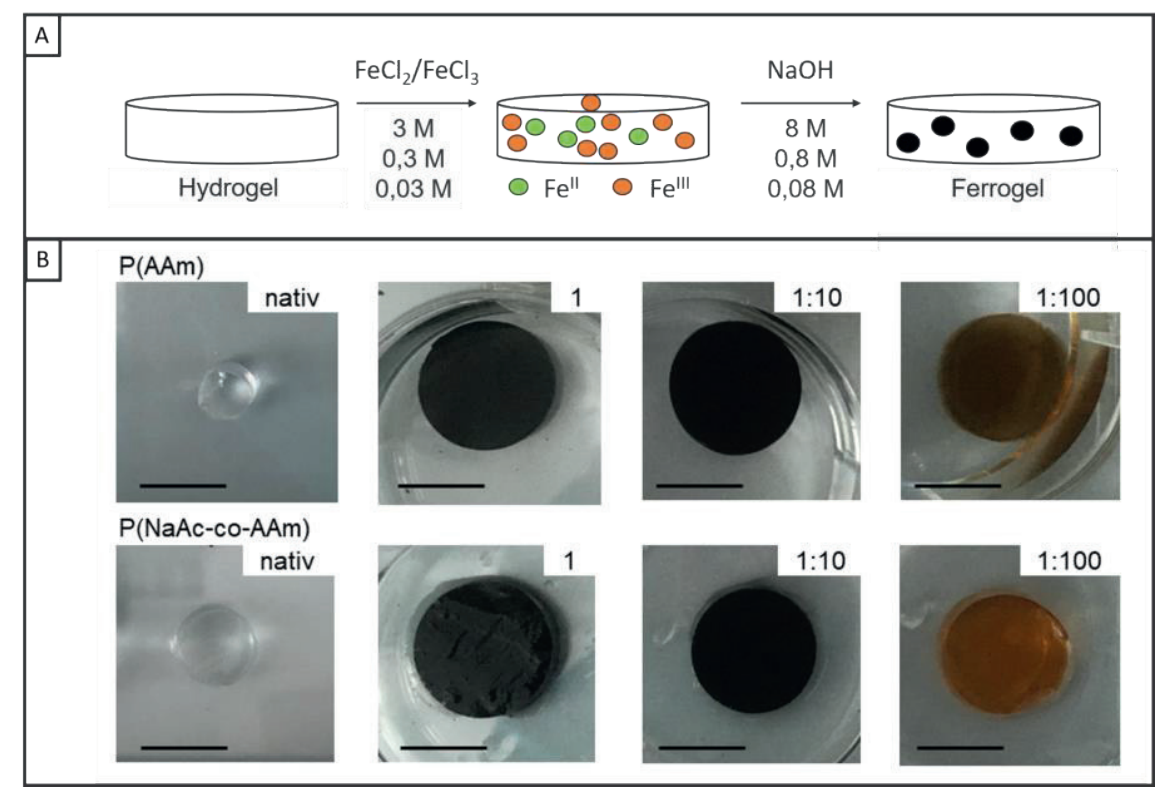

Abb. 1: (A) Übersicht der verwendeten Konzentrationen an Eisensalz- und NaOH-Lösungen zur in-situ Präzipitation von Eisenoxidpartikeln in Hydrogelen. (B) Darstellung der P(AAM) und P(NaAc-co-AAm) Hydrogele sowie der resultierenden Ferrogele in Abhängigkeit von den verwendeten Konzentrationen im Präzipitationsansatz (Maßstabsbalken entspricht $1 \mathrm{~cm}$ ). 


\section{Methoden und Materialien}

\section{Synthese der Hydrogele}

Für die Synthese der P(AAm) Hydrogele nach [6] wurden 1,6 mol/L des Monomers Acrylamid und 1,5 mol-\% des Vernetzers N,N'-Methylen-bis-acrylamid in deionisiertem Wasser gelöst und auf Eis gekühlt. Zur Initiierung der freien radikalischen Polymerisation werden 0,27 mol- $\%$ Ammoniumperoxodisulfat und 2,1 mol-\% $N, N, N^{*}, N^{*}$-Tetramethylethylendiamin zur Lösung gegeben, resuspendiert, in $1 \mathrm{~mL}$ Glasröhrchen pipettiert und verschlossen über Nacht bei Raumtemperatur zur Polymerisation gelagert.

Für die Synthese der P(NaAc-co-AAm) Hydrogele wurden insgesamt 4,769 mol/L der Monomere Natriumacrylat und Acrylamid in einem Verhältnis von 5:95 eingesetzt und mit 1,0 mol- $\%$ des Vernetzers $N, N$-Methylen-bis-acrylamid in deionisiertem Wasser gelöst und auf Eis gekühlt. Zur Initiierung der freien radikalischen Polymerisation werden 0,09 mol-\% Ammoniumperoxodisulfat und 0,7 mol- $\%$ $N, N, N^{*}, N^{\prime}$-Tetramethylethylendiamin zur Lösung gegeben, resuspendiert und ebenso in $1 \mathrm{~mL}$ Glasröhrchen pipettiert sowie verschlossen über Nacht bei Raumtemperatur zur Polymerisation gelagert.

Das Volumen der jeweiligen Syntheseansätze betrug $10 \mathrm{~mL}$. Die polymerisierten Hydrogele wurden aus den Glasröhrchen entfernt und für 5 Tage in deionisiertem Wasser unter mehrmaligem Wechsel gewaschen. Von den zylinderförmigen Hydrogelen wurden ca. $2 \mathrm{~mm}$ dicke Scheiben angefertigt und vor der in-situ Fällung in mehreren Zyklen $1 \mathrm{M}$ Natriumchloridlösung $(\mathrm{NaCl})$ konditioniert, um reproduzierbare Ferrogele zu erhalten.

\section{In-situ Fällung der Eisenoxidpartikel im Hydrogel}

Zur Vorbereitung der Fällung wurden die Hydrogelscheiben mit Stickstoff in deionisiertem Wasser sauerstofffrei gespült und der Fällungsansatz vollständig in einer Stickstoff-gefluteten Handschuhbox (Sauerstoffkonzentration $<0,01 \%$ ) durchgeführt.

Die $3 \mathrm{M}$ Eisenchloridlösung besteht aus Eisen(III)chlorid-Hexahydrat und Eisen(II)-chlorid-Tetrahydrat in einem molaren Verhältnis von 2:1. Von dieser Lösung wurden eine 1:10 und eine 1:100 Verdünnung hergestellt. Im ersten Schritt der Fällung wurde das native Hydrogel in je $5 \mathrm{~mL}$ der entsprechend konzentrierten Eisenchloridlösung für $24 \mathrm{~h}$ gelegt.

Zur Fällung von Eisenoxid wurde eine $8 \mathrm{M}$ Natriumhydroxidlösung und die jeweiligen 1:10 und 1:100 Verdünnungen hergestellt. Die überschüssige Eisensalzlösung wurde abgesaugt und je $5 \mathrm{~mL}$ der entsprechend konzentrierten $\mathrm{NaOH}$-Lösung auf die Eisensalz-getränkten Hydrogele gegeben. Es erfolgte eine Lagerung des Fällungsansatzes über
Nacht. Abschließend wurden die Ferrogele bis zur $\mathrm{pH}-$ Neutralität des Wassers mit deionisiertem Wasser gewaschen.

Die folgenden Experimente zur Charakterisierung der Ferrogele wurden jedoch unter Umgebungsbedingungen durchgeführt, so dass eine Oxidation von Magnetit $\left(\mathrm{Fe}_{3} \mathrm{O}_{4}\right)$ zu Maghämit $\left(\mathrm{y}-\mathrm{Fe}_{2} \mathrm{O}_{3}\right)$ anzunehmen ist.

\section{Bestimmung des Quellgrades}

Um die Stimulus-abhängigen Quelleigenschaften der Hydrogele und Ferrogele zu bestimmen, wurde jeweils die Masse der Proben ohne $\left(m_{0}\right)$ und mit Stimulus $\left(\mathrm{m}_{\mathrm{i}}\right)$ nach je $24 \mathrm{~h}$ ermittelt und der Quellgrad über folgenden Zusammenhang bestimmt:

$$
\text { Quellgrad }[\%]=\frac{m_{i}-m_{0}}{m_{0}} * 100 \%
$$

\section{Ergebnisse}

Abb. 1B zeigt die transparenten Hydrogele sowie die korrespondierenden Ferrogele, welche nach der in-situ Fällung in Abhängigkeit der eingesetzten Verdünnungen schwarz und hellbraun erscheinen. Weiterhin fällt eine deutliche Volumenzunahme der Ferrogele gegenüber den Hydrogelen auf. In Abhängigkeit der verwendeten Konzentration des Präzipitationsansatzes kann auf einen hohen (schwarz) bis niedrigen Gehalt (hellbraun) an Eisenoxidpartikeln im Hydrogel geschlossen werden.

\section{lonensensitivität der Ferrogele}

In Abb. 2 ist der Quellgrad der Hydrogele und Ferrogele in einem Bereich von $1 \mathrm{nM}$ bis $1 \mathrm{M} \mathrm{NaCl}$ aufgetragen. Erwartungsgemäß gibt es eine leichte Quellung von ca. $10 \%$ des neutralen P(AAm) und eine sprunghafte Entquellung von ca. $40 \%$ des anionischen Copolymers in $1 \mathrm{M} \mathrm{NaCl}$.

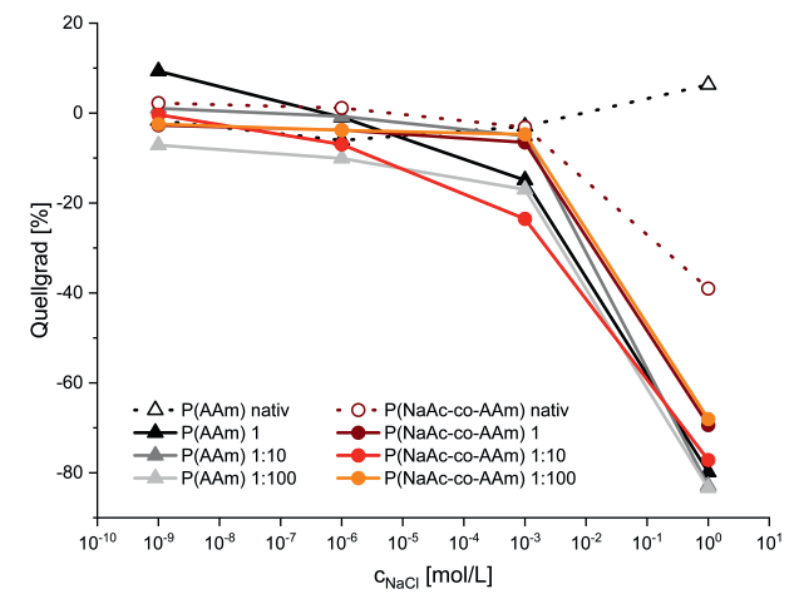

Abb. 2: Quellgrad der Hydrogele und Ferrogele gegenüber $\mathrm{NaCl}$-Lösung verschiedener lonenstärke. 
Ebenso zeigen alle Ferrogele eine sprunghafte Entquellung in $1 \mathrm{M} \mathrm{NaCl}$ mit einem nahezu doppelten Quellgrad von ca. -80 \%. Darüber hinaus entquellen Ferrogele, die aus verdünnten Fällungsansätzen hergestellt wurden, bereits bei lonenstärken im nMund $\mu \mathrm{M}$-Bereich, sodass eine Steigerung der Sensitivität erzielt werden konnte.

\section{pH-Sensitivität der Ferrogele}

Die pH-abhängigen Quellkurven in Abb. 3 zeigen vor allem für die Ferrogele aus den Verdünnungsstufen eine nahezu lineare Quellgradänderung von pH 2 zu pH 7 um 20 bis $40 \%$. Bei pH 8 zeigt sich nochmal eine deutliche Steigerung oder teilweise eine geringe Abnahme des Quellgrades.

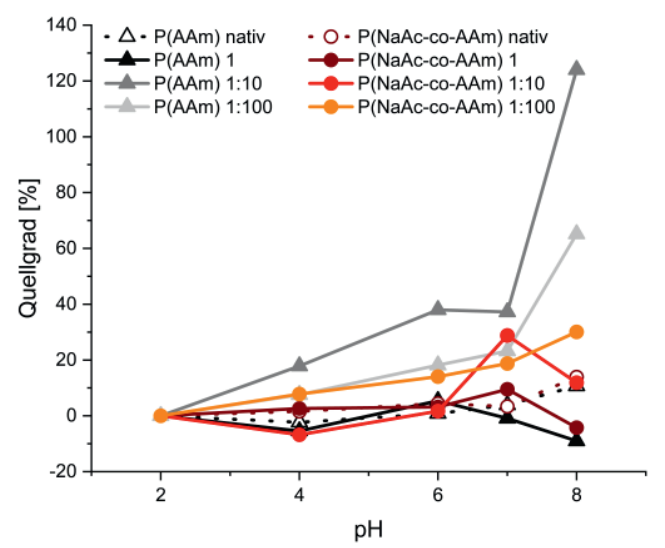

Abb. 3: pH-abhängiger Quellgrad der Hydrogele und Ferrogele.

\section{Kinetik der Ferrogele}

Wie Abb. 4 zeigt, ist auch die Ansprechzeit der Ferrogele gegenüber den Hydrogelen während der Entquellung deutlich gesteigert.

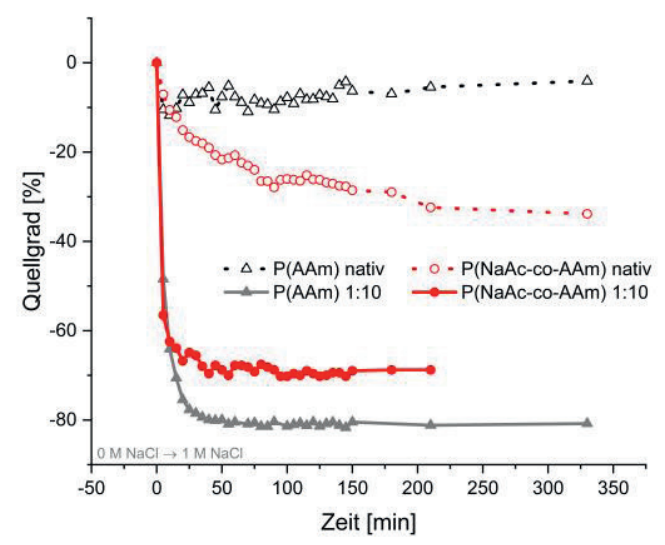

Abb. 4: Kinetik der Entquellung der Hydrogele und Ferrogele in $1 \mathrm{M} \mathrm{NaCl}$.
So erreichen die Hydrogele einen quasi-stationären Quellgrad nach über drei Stunden und die Ferrogele aufgrund der sprunghaften Entquellung bereits nach etwa 30 min.

\section{Diskussion}

Der Quellmechanismus neutraler und lonen-sensitiver Hydrogele beruht auf unterschiedlichen Wechselwirkungen mit der umgebenden lonen-haltigen Lösung. Neutrale Hydrogele quellen unter erhöhter lonenstärke, bis der osmotische Druck der beiden Systeme Hydrogel und Umgebung ausgeglichen ist. Ionen-sensitive Hydrogele mit festgebundenen anionischen Acrylat-Gruppen (pKs-Wert: 4,2) liegen im neutralen $\mathrm{pH}$-Bereich deprotoniert vor [7]. Die dadurch verursachten negativen Ladungen führen zu elektrostatischen Abstoßungen innerhalb des Polymernetzwerkes, sodass dieses im gequollenen Zustand vorliegt. Kommt es zur Diffusion von gelösten Ionen werden die gebundenen Ladungen neutralisiert und das Hydrogel entquillt.

Damit lassen sich zwei Effekte der durchgeführten Quellexperimente erklären:

(1) Die Ionen-sensitive Entquellung und $\mathrm{pH}$-abhängige Quellung aller Ferrogele ist auf eine hohe Dichte an Oberflächen-aktiven Hydroxylgruppen der präzipitierten Eisenoxidpartikel zurückzuführen [8] bzw. kann auch davon ausgegangen werden, dass nicht ein reines Oxid, sondern ein Eisenoxidhydroxid entstanden ist [9]. Je nach pH-Wert besitzen die Eisenoxidpartikel entweder eine protonierte oder eine deprotonierte Oberflächenladung [10]. Der Quellgrad bei pH 7 zeigt, dass bei neutralem $\mathrm{pH}-$ Wert deprotonierte Oberflächenladungen vorliegen (Abb. 3), die wiederrum unter erhöhter lonenstärke neutralisiert werden und damit zur lonensensitiven Entquellung der Ferrogele führen (Abb. 2). Weiterhin verdeutlichen die Quellexperimente, dass vor allem Ferrogele aus geringer konzentrierten Präzipitationsansätzen linear verlaufende Quellkurven besitzen und damit besser als sensorisches Material in Drucksensoren geeignet sind.

(2) Ein geringerer Quellgrad der Copolymer-basierten Ferrogele gegenüber den $\mathrm{P}(\mathrm{AAm})$-basierten Ferrogele kann auf eine zusätzliche Vernetzung der anionischen Copolymere durch die Eisenionen zurückgeführt werden. In der Literatur wird beschrieben, dass Eisenionen in Hydrogelen durch festgebundene, negativ geladene Gruppen koordiniert werden und damit eine zusätzliche physikalische Vernetzung der Hydrogelstruktur bewirken [11]. Der Effekt der physikalischen Vernetzung anionischer Hydrogele durch Eisenionen führt zur Verringerung der Elastizität des Hydrogels und könnte somit den geringeren Quellgrad des Copolymer-basierten Ferrogels erklären. 
Zusammenfassend konnten Stimuli-responsive Ferrogele mittels in-situ Fällung hergestellt werden. Die Ferrogele besitzen eine lonen-sensitive Entquellung bei neutralen $\mathrm{pH}-$ Werten über einen weiten Bereich variierender lonenstärke. Vor allem Ferrogele aus verdünnten Präzipitationsansätzen weisen ein lineares Quellverhalten, eine kurze Ansprechzeit und einen hinreichend hohen Quellgrad auf. Damit erfüllen sie die Anforderungen, um als sensorisches Material im Drucksensor verwendet werden zu können.

\section{Literatur}

[1] P. Devi, A. Thakur, R. Y. Lai, S. Saini, R. Jain, and $P$. Kumar, "Progress in the materials for optical detection of arsenic in water," TrAC Trends Anal. Chem., vol. 110, pp. 97-115, Jan. 2019.

[2] D. E. Giles, M. Mohapatra, T. B. Issa, S. Anand, and $P$. Singh, "Iron and aluminium based adsorption strategies for removing arsenic from water," J. Environ. Manage., vol. 92, no. 12, pp. 3011-3022, 2011.

[3] M. J. Uddin and Y. K. Jeong, "Review: Efficiently performing periodic elements with modern adsorption technologies for arsenic removal," Environ. Sci. Pollut. Res., vol. 27, no. 32, pp. 39888-39912, 2020.

[4] M. Guenther, T. Wallmersperger, and G. Gerlach, "Piezoresistive chemical sensors based

\section{Danksagung}

Die Autoren danken Alexander Kutscher vom Institut für Halbleiter- und Mikrosystemtechnik der TU Dresden für die Bereitstellung der Stickstoff-gespülten Handschuhbox. Weiterhin gilt der Dank der Deutschen Forschungsgemeinschaft (DFG) für die on functionalized hydrogels," Chemosensors, vol. 2, no. 2, pp. 145-170, 2014.

[5] M. Guenther and G. Gerlach, "Hydrogels for Chemical Sensors," 2009, pp. 165-195.

[6] J. Erfkamp, M. Guenther, and G. Gerlach, "Hydrogel-based sensors for ethanol detection in alcoholic beverages," Sensors (Switzerland), vol. 19, no. 5, 2019.

[7] M. J. Krafcik and K. A. Erk, "Characterization of superabsorbent poly(sodium-acrylate acrylamide) hydrogels and influence of chemical structure on internally cured mortar," undefined, vol. 49, no. 11, pp. 4765-4778, Nov. 2016.

[8] E. Darezereshki, A. khodadadi Darban, M. Abdollahy, and A. Jamshidi-Zanjani, "Influence of heavy metals on the adsorption of arsenate by magnetite nanoparticles: Kinetics and thermodynamic," Environ. Nanotechnology, Monit. Manag., vol. 10, no. January, pp. 51-62, 2018.

[9] J. P. Jolivet, C. Chanéac, and E. Tronc, "Iron oxide chemistry. From molecular clusters to extended solid networks," Chem. Commun., vol. 4, no. 5, pp. 477-483, 2004.

[10] W. Stumm, "The Inner-Sphere Surface Complex," pp. 1-32, 1995.

[11] S. Kim, A. U. Regitsky, J. Song, J. llavsky, G. H. McKinley, and N. Holten-Andersen, "In situ mechanical reinforcement of polymer hydrogels via metal-coordinated crosslink mineralization," Nat. Commun., vol. 12, no. 1, pp. 1-10, 2021.

finanzielle Unterstützung im Rahmen des Graduiertenkollegs „Hydrogel-basierte Mikrosysteme“ (GRK 1865). 